\title{
Correlated Knowledge: an Epistemic-Logic View on Quantum Entanglement
}

\author{
Alexandru Baltag • Sonja Smets
}

Received: 25 November 2008 / Accepted: 15 June 2010 / Published online: 30 July 2010

(C) The Author(s) 2010. This article is published with open access at Springerlink.com

\begin{abstract}
In this paper we give a logical analysis of both classical and quantum correlations. We propose a new logical system to reason about the information carried by a complex system composed of several parts. Our formalism is based on an extension of epistemic logic with operators for "group knowledge" (the logic GEL), further extended with atomic sentences describing the results of "joint observations" (the logic LCK). As models we introduce correlation models, as a generalization of the standard representation of epistemic models as vector models. We give sound and complete axiomatizations for our logics, and we use this setting to investigate the relationship between the information carried by each of the parts of a complex system and the information carried by the whole system. In particular we distinguish between the "distributed information", obtainable by simply pooling together all the information that can be separately observed in any of the parts, and "correlated information", obtainable only by doing joint observations of the parts (and pooling together the results). Our formalism throws a new light on the difference between classical and quantum information and gives rise to an informational-logical characterization of the notion of "quantum entanglement".
\end{abstract}

Keywords Logics for quantum information - Quantum correlations · Entanglement · Correlation models · Epistemic logic $\cdot$ Correlated knowledge

\footnotetext{
A. Baltag

Computing Laboratory, Oxford University, Oxford, UK

e-mail: Alexandru.Baltag@ comlab.ox.ac.uk

S. Smets $(\bowtie)$

Dept. of Artificial Intelligence and Dept. of Philosophy, University of Groningen, Groningen, Netherlands

e-mail: S.J.L.Smets@rug.nl

S. Smets

IEG, Oxford University, Oxford, UK
} 


\section{Introduction}

This paper forms part of the recent research trend aimed at connecting the Logical Foundations of Quantum Physics to fundamental issues in Logic and Theoretical Computer Science, and in particular to theories of distributed computation and quantum information (see e.g. $[2,3,5-8,16,18,20])$. Instead of starting with traditional "quantum logic", we focus on one of the logical formalisms (namely, Epistemic Logic) that was successfully used in Theoretical Computer Science. Because of their spatial features, epistemic logics have traditionally been used to model the flow of classical information in distributed systems, including secure communication protocols, distributed computation and multi-agent robotic systems in Artificial Intelligence. Our aim is to extend this domain of applications to include quantum information flow. We thus adapt and generalize epistemic logic to reason about multi-partite quantum systems and quantum correlations.

Our main focus in this paper is the relationship between the information carried by a complex system and the information carried by each of the parts of the system. As examples of complex systems, we look at any physical system composed of more elementary subsystems (particles), but also at any group of "agents" that can perform observations (of their local environment), logical inferences or introspective reflection. The "parts" of a complex system do not have to be independent, on the contrary their behavior (e.g. the agents' observations) might happen to be correlated, either due to prior communication or to other causes (e.g. quantum entanglement). As a result, the pieces of information carried by the parts might not be independent either. Different types of complex systems will manifest different types of correlations or dependencies between the information carried by their parts.

We are interested in modeling these various types of complex systems from an operational point of view. That is to say, we look for the operational criteria that characterize various types of correlations between the local parts of a given system. What we show is that there are several ways in which the information carried by the individual parts of a system can be combined. Depending on how the information between the parts is pooled together, the system will exhibit different types of correlations. Our study of informational correlations will allow us to mark the difference between classical and quantum correlations.

To formalize these notions, we use a version of epistemic logic, in which the epistemic "agents" are to be identified with the elementary parts (basic "subsystems", or "locations") of a complex physical system. As is standard practice in Computer Science, we adopt the "external" view on knowledge advocated by Halpern et al. [21], which allows for applications of epistemic logic to situations that reach beyond the traditional study of real agents' "knowledge": in this view, any of the localized parts of a complex system can be seen as a "virtual agent", while the system itself as well as any of its subsystems can be seen as a virtual "group of agents". The "implicit knowledge" that (is not necessarily actually "possessed" by, but) can be "externally" attributed to such a virtual agent is given simply by the information that is potentially available at the corresponding location: something is "known" if it is a consequence of features that can in principle be observed at that location. Implicit knowledge thus gives us the information (about the overall system) that is carried by a part (of that system). In this sense, we can think of epistemic logic as a "spatial logic", meant to express the relationships between (the information available at) different locations in a complex system. Usually, when considering logics with spatial features, one thinks about a topology or even a metric space, but in this paper we are only interested in the "local" features indicating which information is carried by which part of the system. The relation between systems and their subsystems is what is relevant for us, and not any sense of "distance" or nearness between systems. 
A natural question raised in the epistemic logic literature is the following: is there a sense in which one can externally assign knowledge to a group of (virtual) agents? In other words: what is the implicit knowledge of a group? The relevance of this question to our inquiry comes from the fact that, in our setting, implicit "group knowledge" would capture the information carried by a complex physical system. The standard answer to this question in the current literature is given by distributed knowledge: this is the information obtainable by pooling together (and closing under logical inference) the "knowledge" of each of the "parts" (agents). According to this view, the implicit knowledge of a group is the same as its distributed knowledge; in other words, the information carried by a complex system is nothing but the "sum" of the information carried by its parts.

Our main claim is that, while this standard answer is adequate for classical physics, it fails for quantum systems. An entangled system carries more information than the sum of its parts. Moreover, there are also examples of social situations in which this standard answer fails for real-life agents: whenever a group of agents can cooperate to make joint observations, the implicit knowledge of the group (defined, as for a single agent, in terms of what follows from the features that can in principle be observed by the group) will typically go beyond distributed knowledge (which only takes into account the results of separate, uncorrelated observations by each of the members of the group). Hence, to correctly model the information implicitly carried by a complex system or group of agents, we propose the notion of "correlated knowledge", that takes into account the correlations between the pieces of information carried by the parts of the system, or the potential results of joint observations that can be performed by the group.

For our investigation of group knowledge, we propose a logical system, called "General Epistemic Logic" (GEL), based on an extension to groups of traditional epistemic logic and on a generalization of the usual notion of epistemic model. In Sect. 3, we give a sound and complete axiomatization for GEL. In subsequent sections, we show that correlated knowledge is useful to capture the non-classicality of a physical system (or the existence of correlations between the agents' observational capabilities in a group). We use the logic GEL to give an informational-logical characterization of the properties of "separability" and "entanglement".

In the final section of this paper we extend the logic GEL with operators that make explicit the agents' observational capabilities, obtaining a "Logic of Correlated Knowledge" LCK. We introduce a notion of "correlated models", that generalizes the well-known representation due to Halpern et al. [21] of epistemic models as vector models. We give a sound and complete axiomatization for $\mathbf{L C K}$ with respect to correlated models.

\section{Background Notions}

Information. In this paper we are mainly concerned with the "qualitative" ("logical", or "semantic") aspects of information. In contrast to the syntactic (or quantitative) approach to information, which is primarily concerned with quantitative measures of information (be it in terms of Shannon entropy or von Neumann entropy), the semantic approach is standard in Logic and Computer Science and focuses on the meaning or "aboutness" of information. Semantic information can be true or false and is always "about" something: propositions have a meaning or an "information content". The main issue in this semantic approach is to find the formal laws governing information flow of a specific type, so that we can analyze it, reason about it, verify its correctness etc. There are of course several different ways to model and view "semantic information". In [13, 14], two compatible views are being discussed: the 
first looks at "information as range" and the second at "information as correlation". The first is tied to a possible world model, where an increase in information is related to a decrease of the range of worlds that are considered possible and vice versa (see [4, 13]). Indeed, "Information as range" takes the point of view of an "external observer", whose information-state is represented only via the relations between the worlds that he or she considers possible. The characteristic feature of the second notion, "information as correlation", is the idea that information can manifest itself as a correlation between different situations or their states of affairs. Here, an increase in information is related to a decrease, restriction, or constraint, on the number of possible correlations between different situations. The ideas behind "information as correlation" trace back to Situation theory $[11,19]$ and originated as a tool to capture the "aboutness" or the semantic content of information even in the absence of observers. As a third way to study semantic information, we mention that it is possible to combine the "information as range" and "information as correlation" views in one model. As an example we mention here the epistemic constraint logic in [13] and the interpreted systems in [21]. Also our general epistemic logic in this paper will address issues that are of concern when we look at "information as range" and at "information as correlation".

Implicit Knowledge. We adopt the distinction in the CS literature between implicit and explicit knowledge (see e.g [21]), and use the notion of "information carried by a system" interchangeably with the notion of "implicit knowledge" (in line also with [8, 10]). Explicit knowledge is the actual information possessed by a real agent, the information stored in its "database" or its subjective internal state. Explicit knowledge is not necessarily introspective and might not be closed under logical consequence. In contrast, implicit knowledge can be visualized as what a virtual agent could in principle come to "know" by performing observations on that system and deriving logical consequences. It is the potential knowledge of the agent (what follows implicitly from his possible observations). This is an "external" sense of knowledge: it is the "knowledge" that "we" externally assign to an agent (or a piece of hardware, a particle or even just a spatial location), based on what that agent could observe (or what is in principle observable at that location). Implicit knowledge thus embodies an objective concept of "locally available (semantic) information", and has nothing to do with some subjective state internal to the subsystem: it sums up all information that is potentially available at that location.

As far as implicit knowledge is concerned, there are no problems with positive and negative introspection nor with logical omniscience. Indeed, it seems reasonable to say that if a system carries the information $P$ then it implicitly carries the information that $P$ is true, and that all consequences of $P$ are true, and that the system carries the information $P$. In "agent" terms, this means assuming that the capabilities of our virtual agent include, not only observations, but also logical inferences and acts of reflection (introspection). So implicit knowledge can be assumed to be always truthful and closed under logical inference and under (positive and negative) introspection. In other words, implicit knowledge satisfies the axioms of the modal system known as S5.

\section{General Epistemic Logic}

Consider a complex system composed of $n$ basic components (or "locations"). We denote each basic component with a label from a given (finite) set $N=\{1, \ldots, n\}$. Note that the information carried by this complex system may be distributed throughout space, it can be localized, concentrated at specific spatial "locations" or "components" of the system. 
Hence, some information is potentially available only at some locations, but not at others. We also want to consider (the information) carried by subsystems composed of several (but not necessarily all) components. To capture these spatial features we introduce a generalized "epistemic logic" as a special type of spatial logic, allowing us to have epistemic operators ("knowledge") for both individuals ("agents") and groups (of agents). We use the notion of component and agent interchangeably: metaphorically, imagine associating to each subsystem a virtual agent that can "observe" only the state of that subsystem. In the case of a physical system, this sums up all the information that is obtainable by performing local measurements on that subsystem (and closing under logical inference). We use sets $I \subseteq N$ of labels to denote complex subsystems (or groups of "agents"). The largest group is the "whole world" $N$, while the smallest groups are singletons $\{i\}$ consisting of an individual agent. Our logic will have an "information" (or "implicit knowledge") operator $K_{I}$ for each subsystem I. As mentioned above, knowledge ("episteme") is used here only in the implicit, external sense, as "information that is in principle available" (via local observations) at a given location. So one may think of the proposition $K_{I} P$ as saying that the subsystem, or group, $I$ (potentially) carries the information that $P$ is the case. For groups $\{i\}$ of one individual agent, we use the simplified notation $K_{i}$ instead of $K_{\{i\}}$. The use of $K_{I}$-operators to capture qualitative spatial features of complex systems, extends our previous approach in [8] which in its turn is based on $[5,7]$ and inspired by $[2,16]$.

Observational Equivalence. Let $s$ and $s^{\prime}$ be two possible states of the world (or "possible worlds"), if the implicit information carried by a system I is the same in these two states we write $s \stackrel{I}{\sim} s^{\prime}$, and say that the states are "observationally equivalent", or "indistinguishable”, for system $I$. Again, we simply write $s \stackrel{i}{\sim} s^{\prime}$ when $I=\{i\}$. Observational equivalence for $I$ means in agent's terms that the virtual agent or group of agents (associated to) $I$ can make exactly the same observations (at location $I$ ) in two states of the world. We will use the relation of observational equivalence to give an interpretation to the $K_{I}$-modalities in our logic:

General Epistemic Frame. For a given set $N$ of basic components, a set of states (or "possible worlds") $\Sigma$, a family of binary relations $\{\stackrel{I}{\sim}\}_{I \subseteq N} \subseteq \Sigma \times \Sigma$ for every subsystem $I \subseteq N$, we define a general epistemic frame to be a Kripke frame (or multi-modal frame) $\left(\Sigma,\{\stackrel{I}{\sim}\}_{I \subseteq N}\right)$ subject to the following four conditions:

1. all $\stackrel{I}{\sim}$ are labeled equivalence relations (for every set $I \subseteq N$ );

2. Information is Monotonic w.r.t. groups: if $I \subseteq J$ then $\stackrel{J}{\sim} \stackrel{I}{\sim}$;

3. Observability Principle: if $s \stackrel{N}{\sim} s^{\prime}$ then $s=s^{\prime}$.

4. Vacuous Information: $s \stackrel{\emptyset}{\sim} s^{\prime}$ for all $s, s^{\prime} \in \Sigma$.

We read $s \stackrel{I}{\sim} s^{\prime}$ as: subsystem I cannot distinguish state s from $s^{\prime}$ (via any local observations, performable on $I$ ). In other words, $\stackrel{I}{\sim}$ gives us a notion of "observational equivalence" relative to subsystem $I$. The above conditions seem natural for this interpretation. The first condition says that the relation is reflexive, transitive and symmetric. The second condition assumes that every observation that can be performed by an agent of a group is in principle available to the whole group. In other words, the members of a group have the capacity to share information among themselves. In terms of systems we then say that if a subsystem carries the information that $P$ then the whole system carries the information that $P$. In 
logical terms this captures the fact that information behaves monotonically with respect to group inclusion. And relationally, it says that the states of the world that are observationally equivalent for a system/group $I$ are also observationally equivalent for any subgroup $J \subseteq I$. The third condition says that if two possible states of the world are indistinguishable with respect to the "whole world" $N$ then they are the same. We call this the "observability" principle, as it identifies states of the world that differ in ways that are not observable even by the whole world. Finally, the last condition says that the empty group has no non-trivial information whatsoever: being unable to make any observations, the empty group cannot distinguish between any two states.

Local Information State. Given a general epistemic frame $\left(\Sigma,\{\stackrel{I}{\sim}\}_{I \subseteq N}\right)$, we construct the notion of an $I$-local state, for each subsystem $I \subseteq N$ :

$$
s_{I}:=\left\{s^{\prime} \in \Sigma: s \stackrel{I}{\sim} s^{\prime}\right\}
$$

Here, $s_{i}$ captures the "agent $i$ 's local state of information", while $s_{I}$ does the same for groups.

$\Sigma$-Propositions and Models. Given a general epistemic frame $\left(\Sigma,\{\stackrel{I}{\sim}\}_{I \subseteq N}\right)$, a $\Sigma$-proposition is any subset $P \subseteq \Sigma$. Intuitively, we say that a state $s$ satisfies the proposition $P$ if $s \in P$. We define a general epistemic model to be a structure $\Sigma=\left(\Sigma,\{\stackrel{I}{\sim}\}_{I \subseteq N},\|\|.\right)$, consisting of a general epistemic frame together with a valuation map $\|\|:. \Omega \rightarrow P(\Sigma)$, mapping every element of a given set of atomic sentences into $\Sigma$-propositions. We use the standard notation for satisfaction of atomic sentences in a given state of model $\Sigma$ denoted by $s \models p$ or $s \in\|p\|$. For every model $\Sigma$, we have the usual Boolean operators on $\Sigma$-propositions: $P \wedge Q:=P \cap Q, P \vee Q:=P \cup Q, \neg P:=\Sigma \backslash P, P \Rightarrow Q:=\neg P \vee Q$. We also have the constants $\top_{\Sigma}:=\Sigma$ and $\perp_{\Sigma}:=\emptyset$. Finally, the "knowledge" operator is defined on $\Sigma$ propositions by putting $K_{I} P:=\{s \in \Sigma: t \in P$ for every $t \stackrel{I}{\sim} s\}$.

General Epistemic Logic (GEL). The language of the logic GEL has the following syntax, starting from atomic sentences $p \in \Omega$ :

$$
\varphi:=p|\neg \varphi| \varphi \wedge \varphi \mid K_{I} \varphi
$$

The semantics is given by an interpretation map associating to each sentence $\varphi$ of GEL a proposition $\|\varphi\|$. Equivalently, we extend the satisfaction relation $s \models \varphi$ from atomic sentences to arbitrary formulas $\varphi$. The definition is by induction in terms of the obvious compositional clauses. In particular, for the $K_{I}$ modality we set:

$$
s \models K_{I} \varphi \text { iff } t \models \varphi \text { for all states } t \stackrel{I}{\sim} s .
$$

So $K_{I} \varphi$ is true in a given state $s$, or a system carries the information that $\varphi$ is the case in state $s$, if and only if $\varphi$ holds in all states of the world that are observationally equivalent for $I$ to $s$. We would like to stress the fact that this captures the idea that "information is based on potential observations".

Proof System. In addition to the rules and axioms of propositional logic, the proof system of GEL includes:

1. $K_{I}$-Necessitation. From $\vdash \varphi$, infer $\vdash K_{I} \varphi$ 
2. Kripke's Axiom. $\vdash K_{I}(\varphi \Rightarrow \psi) \Rightarrow\left(K_{I} \varphi \Rightarrow K_{I} \psi\right)$

3. Truthfulness. $\vdash K_{I} \varphi \Rightarrow \varphi$

4. Positive Introspection. $\vdash K_{I} \varphi \Rightarrow K_{I} K_{I} \varphi$

5. Negative Introspection. $\vdash \neg K_{I} \varphi \Rightarrow K_{I} \neg K_{I} \varphi$

6. Monotonicity of Group "Knowledge". For $I \subseteq J$, we have $\vdash K_{I} \varphi \Rightarrow K_{J} \varphi$

7. Observability. $\vdash \varphi \Rightarrow K_{N} \varphi$

Using standard results in Modal Correspondence theory (see e.g. [15]), it is easy to show the following:

Theorem 1 The above proof system is sound and complete with respect to general epistemic frames.

Proof Soundness is trivial: rule 1 and axiom 2 hold in any Kripke model; axioms 3,4,5 hold in any model in which $\stackrel{I}{\sim}$ are equivalence relations; axiom 6 holds in any model satisfying the condition that "information is monotonic" (i.e. $\stackrel{J}{\sim} \subseteq \stackrel{I}{\sim}$ ); axiom 7 holds in any model satisfying the Observability Principle. For completeness, let $\varphi_{0}$ be a sentence that is consistent (with respect to the above proof system). We need to show that $\varphi_{0}$ is satisfiable in some general epistemic model. For this, we use the standard "canonical model" construction familiar from Modal Logic [15], in which states are "theories" (= sets of sentences in the language of GEL) that are maximally consistent with respect to the above proof system. Let $\Omega$ be the set of all such maximally consistent theories. We define equivalence relations $\stackrel{I}{\sim}$ on $\Omega$, by putting for every two theories $T, T^{\prime} \in \Omega: T \stackrel{I}{\sim} T^{\prime}$ iff $\forall \varphi\left(K_{I} \varphi \in T \Longleftrightarrow K_{I} \varphi \in T^{\prime}\right)$. For the valuation, we put $\|p\|:=\{T: p \in T\}$. Axioms 3,4 and 5 ensure that $\stackrel{I}{\sim}$ are equivalence relations; axiom 6 ensures that information is monotonic $(\stackrel{J}{\sim} \subseteq \stackrel{I}{\sim}$ for $I \subseteq J)$; axiom 7 ensures that the Observability Principle holds. However, the "Vacuous Information" condition does not hold in $\Omega$. Still, we can ensure it by restricting our model $\Omega$ to the set $\Omega_{0}:=\left\{T \in \Omega: T \stackrel{\emptyset}{\sim} T_{0}\right\}$, where $T_{0}$ is some fixed maximally consistent theory $T_{0} \in \Omega$ such that $\varphi_{0} \in T_{0}$. (The existence of $T_{0}$ is ensured by the Lindenbaum Lemma, as usually.) Since $\stackrel{I}{\sim} \subseteq \stackrel{\emptyset}{\sim}$ (by monotonicity of information), this restriction preserves all the properties of $\stackrel{I}{\sim}$. In addition, it obviously ensures that the "Vacuous Information" condition holds (since $T \stackrel{\emptyset}{\sim} T_{0} \stackrel{\emptyset}{\sim} T^{\prime}$ for all $T, T^{\prime} \in \Omega_{0}$ ). So we obtain a general epistemic model $\boldsymbol{\Omega}_{0}$. Finally, using all our axioms (including rule 1 and axiom 2), we can prove in the usual way a "Truth Lemma", stating that a sentence $\varphi$ holds at a state $T \in \Omega$ iff it belongs to $T$ (seen as a theory): $T \models \varphi$ iff $\varphi \in T$. As a consequence, $\varphi_{0}$ holds at state $T_{0}$ in the model $\Omega_{0}$ (since $\varphi_{0} \in T_{0}$ ), and hence $\varphi_{0}$ is satisfiable.

\section{Distributed Knowledge}

The notion of distributed knowledge was first introduced in the work of Halpern and Moses [22]. In [21] the authors say that a group has distributed knowledge of $\varphi$ if roughly speaking the agents' combined knowledge implies $\varphi$. Or "distributed knowledge can be viewed as what a wise man-one who has complete knowledge of what every member of the group knows-would know." [21, p. 3]. The intuition is that, in addition to making individual observations, the agents of the group can "combine" their knowledge, by sharing all they 
know: they can announce to the group the knowledge obtained by each member on the basis of their separate observations.

Similar as for implicit knowledge, we introduce a modal operator for the distributed knowledge of a group $I$, denoted by $D K_{I}$. This operator $D K_{I}$ can be defined using a "distributed observational equivalence" relation, which is given by the intersection $\bigcap_{i \in I} \stackrel{i}{\sim}$ of all the individual observational equivalence relations. The idea is that two states are indistinguishable for the group if and only if they are indistinguishable for all the members of the group. Distributed knowledge $D K_{I}$ is simply defined as the Kripke modality for $\bigcap_{i} \stackrel{i}{\sim}$, i.e. given by:

$$
s \models D K_{I} \varphi \text { iff } \quad \text { for every state } t \in \Sigma \text {, if } \forall i \in I s \stackrel{i}{\sim} t \text { then } t \models \varphi .
$$

In [21], the authors identify implicit knowledge of a group $I$ with distributed knowledge $D K_{I}$. We doubt however that this identification holds in all situations. Recall that implicit knowledge was explained as what the agent/group could come to know based on potential observations. So the question is: what are, in general, the observational capabilities of a group? Looking at the definition, we see that the use of the intersection of individual observational equivalencies makes sense only if we assume that a group's observations are nothing but observations done by either of the members of the group. But this is in general a highly unreasonable assumption: joint observations by a group are not in general the same as independent observations by each of the members of the group.

In a group whose implicit knowledge is the same as distributed knowledge, each agent can only share with the group the end-result of all her separate, independent observations, but agents are not allowed to correlate (the results of) their observations, in a simultaneous or sequential manner. But according to us, the natural notion of (implicit) group knowledge is something different, something that could be called "correlated knowledge": this is what the group could come to know by performing joint (correlated) observations and sharing the results.

Separability. For system $J \subseteq I$, we say that the system $I$ is $J$-separable in state $s$ if we have $s_{J} \cap s_{I \backslash J}=s_{I}$. A system $I$ is called fully separable in state $s$ if we have $\bigcap_{i \in I} s_{i}=s_{I}$. Full separability means that group I's knowledge in state $s$ is the same as its distributed knowledge, i.e. $s \models K_{I} P$ iff $s \models D K_{I} P$, for all sets $P \subseteq \Sigma$. Note that if system $I$ is fully separable then it is $J$-separable for all $J \subseteq I$, but the converse is false in general. We call a state $I$-entangled if it is not $I$-separable.

Classical Epistemic Frame. A general epistemic frame $\left(\Sigma,\{\stackrel{I}{\sim}\}_{I \subseteq N}\right)$ is called classical if all its states are fully separable, i.e. if it satisfies $\stackrel{I}{\sim}=\bigcap_{i \in I} \stackrel{i}{\sim}$ for all systems $I$. So a frame is classical if and only if any group's "knowledge" in any state (coincides with its distributed knowledge, and hence) can be obtained by pooling together the information of each of its components: $K_{I}=D K_{I}$.

Classically, a group cannot distinguish two states if and only if no member of the group can distinguish them. Note that not only classical or macroscopic systems will satisfy the conditions of a classical epistemic frame. Indeed, one may also encounter classical epistemic frames in the quantum world. This happens when the subsystems are separated.

The Vector Model Representation of Classical Epistemic Models. Classical epistemic frames (models) can be given a "normal form" representation as vector frames (models): 
Fact ([12]) Let, for each $i, \Sigma_{i}:=\left\{s_{i}: s \in \Sigma\right\}$ be the set of all $i$-local states. Every classical epistemic frame $\Sigma$ can be canonically embedded into the Cartesian product $\Sigma_{1} \times \Sigma_{2} \times \cdots \times \Sigma_{n}$, via some embedding $e$ satisfying

$$
s \stackrel{I}{\sim} s^{\prime} \text { iff } e(s)_{i}=e\left(s^{\prime}\right)_{i} \text { for all } i \in I .
$$

To show this, put $e(s):=\left(s_{i}\right)_{i \in N}$, where $s_{i}=\left\{s^{\prime} \in \Sigma: s \stackrel{i}{\sim} s^{\prime}\right\}$ is the $i$-local state of $s$, as defined in the previous section. This vector representation corresponds to another wellknown way to model epistemic logic: the "interpreted systems" representation, in the style of Halpern et al. [21], in which the global states are simply taken to be tuples of local states, with identity of the $i$-th components as the indistinguishability relation $\stackrel{i}{\sim}$. But note that such a representation is not possible in the case of general (non-classical) epistemic frames!

Surprisingly enough, the same validities hold in the class of classical epistemic models as in the class of general epistemic models. This is shown by the following result:

Proposition 1 The proof system of $\boldsymbol{G E L}$ (given in the previous section) is sound and complete with respect to classical epistemic models as well.

The proof is in Halpern et al. [21]: essentially, this is their proof of completeness for epistemic logic extended with distributed knowledge operators.

In conclusion, the language of general epistemic logic $\boldsymbol{G E L}$ cannot distinguish between general epistemic models and the classical ones. In order to be able to distinguish them, we will have to extend the language of GEL to a richer "logic of correlated knowledge" $\mathbf{L C K}$ (Sect. 6).

\section{Quantum "Knowledge”}

A single quantum system can be represented by a state space $\Sigma$ consisting of rays ${ }^{1}$ in a Hilbert space $H$. A quantum system composed of $N$ subsystems $\Sigma_{1}, \ldots, \Sigma_{n}$ is represented by the state space $\Sigma_{1} \otimes \cdots \otimes \Sigma_{n}$ corresponding to the tensor product $H_{1} \otimes \cdots \otimes H_{n}$. Note that this tensor product is much richer than the Cartesian product $\Sigma_{1} \times \cdots \times \Sigma_{n}$ : when the state of a system $s$ is entangled, then it cannot be decomposed as a tuple of local states. So we cannot think of a composed quantum system as a classical epistemic frame. Nevertheless, we will show that they can still be thought of (non-classical) general epistemic frames, in a natural way.

To see this, we consider the following question: what is the "state" of an entangled subsystem $I$ ? For instance, what is the state of component 1 in the binary system $|00\rangle+|11\rangle$ ? As we saw, the answer for $I$-separable states $s=s_{I} \otimes s_{N \backslash I}$ is simply given by the local state $s_{I}$. But we also saw that one cannot talk in any meaningful way about the $I$-local state of an $i$-entangled system. To proceed, we first give the standard QM definition in terms of density operators, then we justify its usefulness for our purposes by looking at the results of local observations.

The State of a Subsystem. If a global system is in state $s$ (thus having an associated density operator $\rho_{s}$ ), then Quantum Mechanics describes the state $s_{(I)}$ of any of its subsystems $I$

\footnotetext{
${ }^{1}$ I.e. vector identified up to a multiplication with a non-zero scalar.
} 
(possibly entangled with its environment $N \backslash I$ in the state $s$ ) by the density operator

$$
s_{(I)}:=\operatorname{tr}_{N \backslash I}\left(\rho_{s}\right) .
$$

In other words, the "state" of subsystem $I$ is obtained by taking the partial $\operatorname{trace} \operatorname{tr}_{N \backslash I}$ (with respect to the subsystem's environment $N \backslash I$ ) of (the density operator associated to) the global state $s$.

Notice that, when the subsystem $I$ is entangled with its environment $N \backslash I$, the above description does not really give us a "state" in the sense of this paper (i.e. a pure state), but a "mixed state". Nevertheless, as an abstract description, it can still give us an indistinguishability relation $\stackrel{I}{\sim}$ on global states: the specific definition of the "state" of a subsystem is not relevant for us in itself, but only the resulting notion of "identity of states" of the given subsystem. This leads us to the following definition:

Observational Equivalence in Quantum Systems. Two quantum states $s, s^{\prime}$ of a global quantum system $N$ are observationally equivalent ("indistinguishable") for a subsystem $I \subseteq N$ if the mixed states of subsystem $I$ are the same in $s$ and $s^{\prime}$. Formally:

$$
s \stackrel{I}{\sim} s^{\prime} \text { iff } \operatorname{tr}_{N \backslash I}\left(\rho_{s}\right)=\operatorname{tr}_{N \backslash I}\left(\rho_{s}^{\prime}\right) .
$$

This indirect definition using density operators may look ad-hoc and unnatural, but it can be justified in terms of what an observer can learn about an entangled subsystem $I$ by observing only that subsystem (so by performing local measurements on I). Indeed, it is known that a mixed state corresponds to a probability measure over pure states, but what is not always well-appreciated is the meaning of the mixed state $s_{(I)}$ describing a (possibly entangled) subsystem:

Quantum I-Equivalence via Local Observations. Provided that we have an unlimited supply of identical $I$-entangled systems in the same (entangled) global state $s$, imagine that the virtual agent associated to $I$ can perform all possible local measurements (in various bases) on (various copies of) subsystem $I$. The agent can also repeat the same tests on different copies and observe the frequency of each result. After many tests, he can approximate the probability of every given result, for each possible local measurement. The list of all these probabilities (for each result of each type of measurement) gives us the "information carried by subsystem $I$ ", or the "information obtainable by local observations at location $I$ ". Two global states $s, s^{\prime}$ are $I$-indistinguishable if all these probabilities are the same in $s$ and $s^{\prime}$, i.e. if the two states behave the same way under $I$-local measurements.

Quantum I-Equivalence via Remote Evolutions. A third way to define observational equivalence is via invariance under changes that do not affect the information carried by subsystem $I$ (see also [8]):

An evolution (unitary map) $U$ is said to be I-remote (or "remote from $I$ ") if it corresponds to applying only a local unitary map on the subsystem $N \backslash I$ (the "non- $I$ " part of the system, also known as $I$ 's "environment"): i.e., if $U$ is of the form $I d_{I} \otimes U_{N \backslash I}$, where $I d_{I}$ is the identity map on subsystem $I$ and $U_{N \backslash I}$ is a unitary map on the subsystem $N \backslash I$. In other words, $U$ is $I$-remote if it is $N \backslash I$-local.

Intuitively, $I$-remote evolutions should not affect the "state" of subsystem $I$; hence, we could define the "state" of $I$ as what is left invariant by all $I$-remote evolutions. As a consequence, two states will be $I$-indistinguishable if they differ only by some $I$-remote evolution. 
The following result shows the equivalence of these three ways of defining observational equivalence:

Proposition 2 For $I \subseteq N$, and $s, s^{\prime} \in \Sigma$, the following are equivalent:

(1) $\operatorname{tr}_{N \backslash I}\left(\rho_{s}\right)=\operatorname{tr}_{N \backslash I}\left(\rho_{s^{\prime}}\right)$;

(2) for every I-local measurement, the probability of obtaining any given result is the same in state $s$ as in state $s^{\prime}$;

(3) $s^{\prime}=U(s)$ for some I-remote unitary map.

So we can define the quantum equivalence relation $s \stackrel{I}{\sim} s^{\prime}$, and hence our notion of implicit knowledge $K_{I}$, by any of the clauses given above. Using for example the third clause we obtain that $K_{I} P$ holds at $s$ iff, for all $I$-remote evolutions $U, P$ holds at $U(s)$. If $P$ is implicitly known by $I$ in state $s$, i.e. if $s \in K_{I} P$, then we say that the subsystem I carries the information that $P$.

A quantum epistemic frame is a (state space $\Sigma$ associated to a) Hilbert space endowed with the quantum $I$-equivalence relations $\stackrel{I}{\sim}$ (as defined above) for every subsystem $I$.

Proposition 3 Quantum epistemic frames are (i.e. satisfy all the postulates of) general epistemic frames.

Properties In addition to the properties of the $K_{I}$ operator in GEL, we add:

- If $s$ is $I$-separable, then $s \stackrel{I}{\sim} s^{\prime}$ iff $s_{I}=s_{I}^{\prime}$

- If $I$ is fully separable then we have: $s \stackrel{I}{\sim} s^{\prime}$ iff $s \stackrel{i}{\sim} s^{\prime}$ for all $i \in I$. As a consequence, the quantum "group" knowledge $K_{I}$ of a fully separated system $I$ is the same as the "distributed knowledge" DK $K_{I}$ of the "group" I.

- In general (for non-fully separated systems $I$ ), the previous statement is false: the information $K_{I}$ carried by a quantum (sub)system $I$ is not the "sum" $D K_{i \in I}$ of the information carried by its $i$-component systems. In other words: quantum epistemic frames are "nonclassical".

Example For instance, in a Bell state when the information stored in two subsystems is correlated according to the identity rule, the agents associated to these subsystems will never recover fully the information possessed by the global system if they cannot correlate the results of their individual observations. Indeed, the following observation shows that in the Bell state $|00\rangle+|11\rangle$ composed of two entangled qubits 1 and 2, the two subsystems 1 and 2 are in the same mixed state:

Proposition 4 The following are equivalent:

1. $s \stackrel{1}{\sim}|00\rangle+|11\rangle$

2. $s \stackrel{2}{\sim}|00\rangle+|11\rangle$

3. $s=|z 0\rangle+\left|z^{\prime} 1\right\rangle$, for some orthogonal vectors $z, z^{\prime} \in H_{1}$.

(The equivalence of e.g. the second and the third clause can be shown by finding a 2-remote evolution $U_{1} \otimes I d_{2}$ such that $U_{1}(|0\rangle)=z$ and $U_{1}(|1\rangle)=z^{\prime}$, and using the definition of $I$ equivalence in terms of remote evolutions.) 
This proposition tells us that, in the state $|00\rangle+|11\rangle$, the two subsystems carry exactly the same information. So pooling together their "knowledge" will not lead to any increase of information: the "distributed knowledge" of the group $\{1,2\}$ is the same as the implicit knowledge of each of the qubits 1 and 2. In contrast, the "group knowledge" of $\{1,2\}$ is much stronger: $s \stackrel{\{1,2\}}{\sim}|00\rangle+|11\rangle$ is equivalent to $s=|00\rangle+|11\rangle$. The group "knows" its own state, so it knows (that the qubits are in) the Bell state $|00\rangle+|11\rangle$.

Informational Characterizations of Separability and Entanglement. Recall that we already gave an "epistemic" characterization of entanglement and separability in general epistemic frames:

A state $s$ is I-separable iff I's knowledge in state $s$ is the same as its distributed knowledge.

In the special case of quantum systems, this gives us the standard Quantum Mechanical notion of separability. ${ }^{2}$ But this characterization cannot be expressed in the language of epistemic logic since it involves a second-order quantifier over all subsets $P$ of the state space: it requires that, for every such subset, $s$ satisfies $K_{I} P$ iff it satisfies $D K_{I} P$.

However, in line with the ideas presented in [8] we can use epistemic logic to give "informational characterizations" of separability and entanglement in a quantum system, provided we are given only one (logical constant denoting a) fully separable state. Indeed, let $w=w_{0} \otimes \cdots w_{n}$ be some (fixed) fully separable state; for example, we may take $w=\overline{0}=|0\rangle^{\otimes N}=|0\rangle \otimes|0\rangle \cdots|0\rangle$. Then we have:

Two subsystems $I$ and $J$ are entangled in a (global) state $s$ iff $s$ satisfies $K_{J} K_{I} \neg w$ or (equivalently, $K_{I} K_{J} \neg w$ ). The state $s$ is $I$-entangled iff the subsystem $I$ and $N \backslash I$ are entangled in $s$, i.e. if $s$ satisfies $K_{I} K_{N \backslash I} s$. The system is separable if it is not entangled: $\neg K_{I} K_{N \backslash I} \neg w$.

To summarize: two physical systems are entangled if and only if they potentially carry (nontrivial) information about each other (assuming no prior communication).

Example For $n=2$, we consider the set $\Sigma_{(1)}$ of all 1-separable (=2-separable $=$ fully separable) global states, as our model. Then given that the system is in state $|00\rangle$, subsystem 1 is in state $|0\rangle$ and "implicitly knows" his own state. 1 implicitly knows also that it is not possible that the whole system is in state $|10\rangle$. Hence, $|00\rangle \models K_{1} \neg|10\rangle$. Subsystem 1 does not implicitly know the local state of the other component when they are fully separable. So subsystem 1 does not implicitly know that the global state is not $|01\rangle$ or in other words: $|00\rangle \models \neg K_{1} \neg|01\rangle$. Subsystem 2 implicitly knows that the global state cannot be $|11\rangle$, however subsystem 1 doesn't know that the local state of subsystem 2 is not $|1\rangle$. Hence subsystem 1 does not know that 2 excludes state $|11\rangle$, that is to say $|00\rangle \models \neg K_{1} K_{2} \neg|11\rangle$.

\section{Correlated Knowledge}

In addition to our presentation so far, our complex systems can be modeled more accurately if we add structure to our general epistemic frames and enrich the language of the logic. In

\footnotetext{
${ }^{2}$ In QM, a separable quantum state refers to a non-entangled state. For instance the global state of a bi-partite system is separable if it belongs to the Cartesian product $H_{1} \times H_{2}$ of the two corresponding Hilbert spaces; in this case, each of the subsystems is in a well-defined ("pure") local state.
} 
this section we will only provide a brief sketch of how this can be brought about, leaving the further details to be explored in future work. The idea is to capture explicitly the "observational capabilities" of the individual agents and of the groups. This allows us to generalize the concrete semantics given by "interpreted systems" (i.e. the vector model representation of classical epistemic models) to a type of general epistemic frames that we call correlation models.

We generalize vector models in three stages: the first type of models we consider are relation-based models. In these models, the states are relations between the agents' possible observations. Given sets $O_{1}, \ldots, O_{n}$ of possible observations for each agent, a joint observation will be a tuple of observations $o=\vec{o}=\left(o_{i}\right)_{i \in N} \in O_{1} \times \cdots \times O_{n}$. A state of the world can be characterized by the joint observations that can be performed on it, so a state is a set of such tuples namely a relation. A model will have as its state space any set $\Sigma \subseteq \mathcal{P}\left(O_{1} \times \cdots \times O_{n}\right)$. The state $s_{I}$ of a subsystem $I$ of a global system in state $s$ will be naturally given by the projection: $s_{i}=\left\{\left(o_{i}\right)_{i \in I}: o \in s\right\}$. So the observational equivalence is then given by: $s \stackrel{I}{\sim} t$ iff $\left\{\left(o_{i}\right)_{i \in I}: o \in s\right\}=\left\{\left(o_{i}\right)_{i \in I}: o \in t\right\}$.

The second, wider stage of generalization is given by multi-set models. Instead of sets of tuples of observations as in the relational models, we now consider multi-sets. Working with multisets has the advantage that we can model the case when agents record the frequencies of their observations. Now the states are multi-sets of joint observations, i.e. functions $s$ from tuples of observations from $O_{1} \times \cdots \times O_{n}$ into natural numbers. The state $s_{I}$ of a subsystem $I$ in a global state $s$ will be naturally given by

$$
s_{I}\left(\left(e_{i}\right)_{i \in I}\right):=\sum\left\{s(o): o \in O_{1} \times \cdots \times O_{n} \text { such that } o_{i}=e_{i} \text { for all } i \in I\right\} .
$$

The third type of models we consider are correlation models. We generalize natural numbers to an abstract set $R$ of possible observational results, together with some abstract operation $\sum: \mathcal{P}(R) \rightarrow R$ of composing results. This operation may be partial (i.e. defined only for some subsets $A \subseteq R$ ), but it is required to satisfy the condition: $\sum\left\{\sum A_{k}: k \in K\right\}=\sum\left(\bigcup_{k \in K} A_{k}\right)$ whenever $\left\{A_{k}: k \in K\right\}$ are pairwise disjoint. In this case, $\left(R, \sum\right)$ will be called a result structure.

Correlation Models. Given a result structure $R$ and a tuple $\vec{O}=\left(O_{i}\right)_{i \in N}$ of sets of possible observations, a correlation model over $\left(R, \sum, \vec{O}\right)$ is given by a set $\Sigma \subseteq\{s: s$ is a function : $\left.O_{1} \times \cdots O_{n} \rightarrow R\right\}$ of maps assigning results to (global) joint observations $o=\left(o_{i}\right)_{i \in N}$. So global states will then be functions from $O_{1} \times \cdots \times O_{n}$ into $R$. We put $O_{I}:=\times_{i \in I} O_{i}=$ $\left\{\left(o_{i}\right)_{i \in I}: o_{i} \in O_{i}\right.$ for every $\left.i \in I\right\}$. As before, in a global state $s$, the state $s_{I}$ of a subsystem $I$ will be a map from $s_{I}: O_{I} \rightarrow R$, given by:

$$
s_{I}\left(\left(e_{i}\right)_{i \in I}\right):=\sum\left\{s(o): o \in O_{1} \times \cdots \times O_{n} \text { such that } o_{i}=e_{i} \text { for all } i \in I\right\} .
$$

To put this more succinctly, for every tuple $e=\left(e_{i}\right)_{i \in I} \in O_{I}$ of $I$-observations, let

$$
\bar{e}:=\left\{o=\left(o_{i}\right)_{i \in N} \in O_{1} \times \cdots \times O_{n}: o_{i}=e_{i} \text { for all } i \in I\right\} .
$$

Then we can define, for every $e \in O_{I}$ :

$$
s_{I}(e)=\sum\{s(o): o \in \bar{e}\} .
$$


Correlated Knowledge. Correlation models are general epistemic models, in which we take our observational equivalence to be identity of the corresponding local states:

$$
s \stackrel{I}{\sim} t \text { iff } s_{I}=t_{I}
$$

The "group knowledge" $K_{I}$ in a correlation model will be called correlated knowledge.

It is easy to see that, in general, correlation models are not necessarily classical (as epistemic frames). Hence, correlated knowledge is in general different from distributed knowledge.

\section{Examples.}

- The relation-based models mentioned first can be recovered as special cases of correlation models, if we take $R=\{0,1\}$ and logical disjunction as the composition operation.

- Epistemic vector models can be seen as special cases of relation-based models (in which every state is a singleton consisting of only one joint observation), and hence they also are correlation models.

- The multi-sets models are also correlation models, with $R$ being the set of natural numbers, and addition as the composition operation.

- Quantum epistemic systems $\Sigma_{1} \otimes \Sigma_{2} \otimes \cdots \otimes \Sigma_{n}$ are correlation models, in which the sets of observations $O_{i}$ are given by the (state spaces associated to) Hilbert spaces $\Sigma_{i}$. Joint observations $\left(o_{i}\right)_{i \in I}$ are interpreted as projectors onto the corresponding state in $\bigotimes_{i \in I} \Sigma_{i}$. The result structure is the interval $R=[0,1]$ with renormalized addition. The "result" of a joint observation $\left(o_{i}\right)_{i \in I}$ made on a state $s$ is interpreted as the probability that the outcome of a local measurement (in any basis that includes $o=\otimes_{i \in I} o_{i}$ ) of the $I$-subsystem of a (global system in) state $s$ will be $o$. It is well-know that any quantum state $s \in \bigotimes_{i \in N} \Sigma_{i}$ is uniquely characterized (up to multiplication by a non-zero scalar) by the function mapping any fully separable state $o=o_{1} \otimes \cdots o_{n} \in \Sigma_{1} \times \cdots \times \Sigma_{n}$ to the probability $|\langle s, o\rangle|^{2}$ of $s$ collapsing to $o$ (after a measurement in a basis that includes $o$ ). ${ }^{3}$

The Logic of Correlated Knowledge. We extend the general epistemic logic GEL with atomic sentences describing the results of possible joint observations by groups of agents, obtaining the logic of correlated knowledge LCK:

$$
\varphi::=p\left|o^{r}\right| \neg \varphi|\varphi \wedge \psi| K_{I} \varphi
$$

where $r \in R$ and $o=\left(o_{i}\right)_{i \in I} \in O_{I}$ is a $I$-tuple of observations, for any subset $I \subseteq N$ of agents. (Recall that $O_{I}:=\times_{i \in I} O_{i}$.) The semantics of $o^{r}$ is naturally given by: $s \models o^{r}$ iff $s_{I}(o)=r$.

Notation For any group $I \subseteq N$, any set $E \subseteq O_{I}$ of $I$-observations and any $E$-tuple of results $r=\left(r_{e}\right)_{e \in E} \in R^{E}$, one for each observation $e \in E$, put

$$
E^{r}:=\bigwedge_{e \in E} e^{r_{e}}
$$

\footnotetext{
${ }^{3} \mathrm{~A}$ different type of relational models for a generalized version of QM is proposed in [17]. Note that our models are "relational" in the sense that quantum "states" correspond in our settings to relations (in relationbased models) or functions (in correlation models). In contrast, in the categorical approach of [17], relations (between finite sets) play the role of morphisms, i.e. they are the analogue of linear maps (between Hilbert spaces) in QM.
} 
Proof System. Fix a finite set $N=\{1, \ldots, n\}$ of agents, a finite result structure $\left(R, \sum\right)$ and a tuple of finite observation sets $\vec{O}=\left(O_{1}, \ldots, O_{n}\right)$. The proof system of LCK over $\left(R, \sum, \vec{O}\right)$ includes the rules and axioms $1-7$ of the logic GEL, and in addition, for every $I \subseteq N$, the following axioms:

8. Observations always yield results: for every $I \subseteq N$, we have

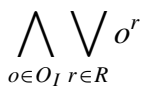

9. Observations have unique results: i.e. for $r \neq p, o \in O_{I}$, we have

$$
o^{r} \Rightarrow \neg o^{p}
$$

10. Groups know the results of their (joint) observations: for $o \in O_{I}, r \in R$, we have

$$
o^{r} \Rightarrow K_{I} o^{r}
$$

11. Group knowledge is correlated knowledge (i.e. is based on joint observations): for every tuple $\left(r_{o}\right)_{o \in O_{I}}$ of results, one for each possible joint observation $o=\left(o_{i}\right)_{i \in I} \in O_{I}$ by group $I$, we have

$$
\left(O_{I}^{r} \wedge K_{I} \varphi\right) \Rightarrow K_{\emptyset}\left(O_{I}^{r} \Rightarrow \varphi\right)
$$

12. Result Composition Axiom: for every tuple $e=\left(e_{i}\right)_{i \in I} \in O_{I}$ of $I$-observations and every tuple $r=\left(r_{o}\right)_{o \in \bar{e}}$, one for each global observation $o \in \bar{e}$, put $\sum r:=\sum\left\{r_{o}: o \in \bar{e}\right\}$; then we have

$$
\bar{e}^{r} \Rightarrow e^{\sum r}
$$

(In axioms 11 and 12 we used the notation $E^{r}$ from above: first with $E=O_{I}$; then with $E=\bar{e}, I=N$.)

Theorem 2 (Soundness and Completeness for LCK) For every finite set $N=\{1, \ldots, n\}$ of agents, every finite result structure $\left(R, \sum\right)$ and every tuple of finite observation sets $\vec{O}=$ $\left(O_{1}, \ldots, O_{n}\right)$, the above proof system is sound and complete with respect to correlation models over $\left(R, \sum, \vec{O}\right)$.

Proof Soundness is trivial: axioms 8 and 9 hold because each state $s$ induces a function $s_{I}: O_{I} \rightarrow R$; axioms 10 and 11 hold because of the definition of indistinguishability in correlation models in terms of the $s_{I}$ functions: $s \stackrel{I}{\sim} t$ iff $s_{I}=t_{I}$; axiom 12 holds due to the specific definition of the map $s_{I}$ (in terms of the map $s$ and of the composition operation $\sum$ ) in correlation models. For completeness, let $\varphi_{0}$ be a consistent sentence. First, we introduce the restricted canonical model $\Omega_{0}$, constructed as in the proof of Theorem 1 (with the same definition of valuation extended to the atomic constants $o^{r}$, i.e. $\left\|o^{r}\right\|:=\left\{T \in \Omega_{0}: o^{r} \in T\right\}$ ). As in Theorem 1, we show that this is a general epistemic model and that it satisfies a "Truth Lemma" ( $T \models \varphi$ iff $\varphi \in T)$, and hence that $\varphi_{0}$ holds at $T_{0}$. Second, we can define a map $T \mapsto s_{T}$, associating to each theory $T \in \Omega_{0}$ some function $s_{T}: O_{1} \times \cdots \times O_{n} \rightarrow R$, given for each $o \in O_{1} \times \cdots \times O_{n}$ by: $s_{T}(o)=r$ iff $o^{r} \in T$. Axioms 8 and 9 (with $I=$ $N=\{1, \ldots, n\}$ ) ensure that the map $T \mapsto s_{T}$ is well-defined, and axiom 11 (with $I=N$, hence with $K_{I} \varphi$ meaning the same as $\varphi$ ) ensures that this map is injective. Hence, we can "identify" theories $T$ with the corresponding functions $s_{T}$, or in other words we can "lift" 
the epistemic model structure from $\Omega_{0}$ to a subset $\left\{s_{T}: T \in \Omega_{0}\right\} \subseteq\{s: s$ is a function : $\left.O_{1} \times \cdots O_{n} \rightarrow R\right\}$, by putting $s_{T} \stackrel{I}{\sim} s_{T^{\prime}}$ iff $T \stackrel{I}{\sim} T^{\prime}$ and $\|p\|=\left\{s_{T}: p \in T\right\}$ (and similarly for the atomic constants $o^{r}$ ). We can easily see that this is a correlation model: axioms 10 and 11 ensure that the indistinguishability condition for correlation models $\left(s \stackrel{I}{\sim} t\right.$ iff $\left.s_{I}=t_{I}\right)$ is satisfied, and axiom 12 ensures that our specific definition (in terms of the map $s$ and of the composition operation $\sum$ ) of the map $s_{I}$ in correlation models is satisfied. As general epistemic models, $\Omega_{0}$ and this correlation model are in fact isomorphic, hence $\varphi_{0}$ is satisfied in (state $s_{T_{0}}$ of) this correlation model.

\section{Concluding Remarks}

In this paper we modeled complex systems (from classical to quantum) using the setting of General Epistemic Frames and we introduced a particular type of such frames called Correlation Models. Our aim has been to throw new light on the difference between classical and quantum information and to gain a better understanding of quantum correlations. As such this paper should be of particular interest to quantum logicians, looking for an abstract formal logical setting that can naturally accommodate entanglement. Note that entanglement posed a problem to the lattice-theoretic approach of traditional Quantum Logic (see e.g. [1, 23]), which opened the road to the quest for possible solutions and alternative settings. Our paper might also be of interest to quantum information theorists who want to abstract away from Hilbert space models, looking for higher levels of abstraction similar to those successfully developed for classical computing. We note that a shorter version of this paper was presented during the 6th QPL workshop on Quantum Physics and Logic held at Oxford University in 2009 [9].

Acknowledgements During the writing of this paper, S. Smets' research was supported by a post-doctoral fellowship of the Flemish Fund for Scientific Research. S. Smets thanks the Flemish Academic Centre for Science and the Arts (VLAC) in Brussels for supporting part of this work by granting her a four-month VLAC-fellowship in 2008. During the writing of this paper, A. Baltag's research was partially supported by the Netherlands Organisation for Scientific Research (NWO), grant number B 62-635, which is herewith gratefully acknowledged. We would also like to kindly thank the anonymous referee for very useful comments.

Open Access This article is distributed under the terms of the Creative Commons Attribution Noncommercial License which permits any noncommercial use, distribution, and reproduction in any medium, provided the original author(s) and source are credited.

\section{References}

1. Aerts, D.: Description of compound physical systems and logical interaction of physical systems. In: Beltrametti, E.G., van Fraassen, B.C. (eds.) Current Issues on Quantum Logic, Ettore Majorana, International Science Series, Physical Sciences, vol. 8, pp. 381-405. Kluwer Academic, Dordrecht (1981)

2. Abramsky, S., Coecke, B.: A categorical semantics of quantum protocols. In: Proceedings of the 19th IEEE Conference on Logic in Computer Science (LiCS'04). Available at arXiv:quant-ph/0402130

3. Abramsky, S., Duncan, R.: A categorical quantum logic. Math. Struct. Comput. Sci. 469-489 (2006)

4. Baltag, A., Moss, L., van Ditmarsch, H.: Epistemic logic and information update. In: Handbook on the Philosophy of Information. Elsevier, Amsterdam (2008)

5. Baltag, A., Smets, S.: The logic of quantum programs. In: Selinger, P. (ed.) Proceedings of the 2nd International Workshop on Quantum Programming Languages (QPL2004). TUCS General Publication, vol. 33, pp. 39-56. Turku Center for Computer Science (2004). PHILSCI00001799 
6. Baltag, A., Smets, S.: Complete axiomatizations of quantum actions. Int. J. Theor. Phys. 44(12), 22672282 (2005)

7. Baltag, A., Smets, S.: LQP: the dynamic logic of quantum information. Math. Struct. Comput. Sci. 16(3), 491-525 (2006)

8. Baltag, A., Smets, S.: A dynamic-logical perspective on quantum behavior. Stud. Log. 89, 185-209 (2008)

9. Baltag, A., Smets, S.: Correlated information: a logic for multi-partite quantum systems. In: Coecke, B., Panangaden, P., Selinger , P.(eds.), Pre-Proceedings of the 6th Workshop on Quantum Physics and Logic, pp. 4-14. Oxford, UK. Electronic Notes in Theoretical Computer Science (2009, forthcoming)

10. Barwise, J.: On the model theory of common knowledge. In: The Situation in Logic. CSLI Lecture Notes, pp. 201-220. Center for the Study of Language and Information (1989)

11. Barwise, J., Perry, J.: Situations and Attitudes. MIT Press, Cambridge (1983)

12. van Benthem, J.: Exploring Logical Dynamics. CSLI Publications, Stanford (1996)

13. van Benthem, J., Martinez, M.: The stories of logic and information. In: Handbook of the Philosophy of Information. Elsevier, Amsterdam (2008). Available at http://dare.uva.nl/record/262024

14. van Benthem, J.: Information as correlation vs. Information as range: a proposal for identifying and merging two basic logical traditions. In: Moss, L. (ed.) In Memory of Jon Barwise (in press). Available at http://www.illc.uva.nl/Publications/ResearchReports/PP-2006-07.text.pdf

15. Blackburn, P., de Rijke, M., Venema, Y.: Modal Logic. Cambridge Tracts in Theoretical Computer Science, vol. 53. Cambridge University Press, Cambridge (2001)

16. Coecke, B.: The logic of entanglement. Research Report, March 2004. arXiv:quant-ph/0402014

17. Coecke, B., Edwards, B.: Toy quantum categories. In: Proceedings of Quantum Physics and Logic 2008. Electronic Notes in theoretical Computer Science (to appear). http://arxiv.org/abs/0808.1037

18. Chiara, M.L. Dalla, Giuntini, R., Leporini, R.: Quantum computational logics. A survey. In: Hendricks, V., Malinowski, J. (eds.) Trends in Logic. 50 Years of Studia Logica, pp. 229-271. Kluwer, Dordrecht (2003)

19. Devlin, K.: Logic and Information. Cambridge University Press, Cambridge (1991)

20. Gudder, S.: Quantum computational logic. Int. J. Theor. Phys. 42(1), 39-47 (2003)

21. Fagin, R., Halpern, J., Moses, Y., Vardi, M.: Reasoning about Knowledge. MIT Press, Cambridge (1995)

22. Halpern, J.Y., Moses, Y.: Knowledge and common knowledge in a distributed environment. J. ACM 37(3), 549-587 (1990)

23. Valckenborgh, F.: Compound systems in quantum axiomatics. Doctoral thesis, Vrije Universiteit Brussel (2001) 